\title{
Cloud Computing Tipping Point Model
}

\author{
Chris Peiris \\ Faculty of Information Sciences and \\ Engineering \\ University of Canberra \\ ACT 2601, Australia \\ $+61414238903$ \\ Chris.Peiris@canberra.edu.au
}

\author{
Bala Balachandran \\ Faculty of Information Sciences and \\ Engineering \\ University of Canberra \\ ACT 2601, Australia \\ $+61262012153$
}

Bala.Balachandran@canberra.edu.au

\author{
Dharmendra Sharma \\ Faculty of Information Sciences and \\ Engineering \\ University of Canberra \\ ACT 2601, Australia \\ $+61262012153$
}

Dharmendra.Sharma@canberra.edu.au

\begin{abstract}
Recently a continuing trend toward IT industrialization has grown in popularity. IT services delivered via hardware, software and people are becoming repeatable and usable by a wide range of customers and service providers. This is due, in part, to the commoditization and standardization of technologies, virtualization and the rise of service-oriented software architectures, and (most importantly) the dramatic growth in popularity/use of the Internet and the Web. Taken together, they constitute the basis of a discontinuity that amounts to a new opportunity to shape the relationship between those who use IT services and those who sell them. The discontinuity implies that the ability to deliver specialised services in IT can be paired with the ability to deliver those services in an industrialised and pervasive way. The reality of this implication is that users of ITrelated services can focus on what the services provide them, rather than how the services are implemented or hosted. Analogous to how utility companies sell power to subscribers, and telephone companies sell voice and data services, some IT services such as network security management, data centre hosting or even departmental billing can now be easily delivered as a contractual service. This notion of cloud computing capability is gathering momentum rapidly. However, the governance and enterprise architecture to obtain repeatable, scalable and secure business outcomes from cloud computing is still greatly undefined. This paper attempts to evaluate the enterprise architecture features of cloud computing and investigates a model that an IT organisation can leverage to predict / evaluate the 'tipping point' where an organisation can make an objective decision to invest in cloud computing. Current research results are attempting to build a quantitative and qualitative service centric framework by mapping cloud computing features with ValIT and COBIT industry best practices.
\end{abstract}

Keywords - cloud computing, could enterprise architecture, cloud computing value model.

\section{INTRODUCTION}

Cloud computing initiatives are gathering significant momentum currently. However, there are no guidelines or models to empower organizations to decide when they should leverage cloud computing. The organisations are aware of the capabilities of cloud computing and benefits it offers. However, organisations do need to radically alter their IT infrastructure, procurement process, management reporting and governance processes to embrace these benefits. Therefore, an organisation is required make significant investment in people, IT and processes to leverage cloud computing benefits. In some cases, this eventuates to significant changes in their business models.
The focus of this paper is to create a model that organisations can leverage to understand whether they arrived at this "crossroad" or "tipping point" to alter their focus from in house IT capability to leveraging cloud computing solutions.

The paper attempts to investigate enterprise architectures for cloud computing capability. Currently, the promise of infinite scalability and abundance of resources under the cloud computing model comes with heavily customised proprietary platforms. These proprietary platforms restrain the users from using specific proprietary software and tie them to a specific software capability. There is a growing need create an open, reusable and scalable enterprise architecture for cloud computing. Therefore, we present a set of high level characteristics for a proposed enterprise architecture for cloud computing that can evolve into an enterprise architecture for cloud computing and summarises further future research extensions.

\section{EVALUTION OF CLOUD COMPUTING ENTERPRISE ARCHITECTURE}

Some of the empirical enterprise architectures are Zachman framework, GERA (Generalized Enterprise Reference Architecture), ITIL (IT Infrastructure Library) and COBIT (Control Objectives for the Information and related Technology). These enterprise architectures are designed to ensure that the businesses are able to get the best out of IT systems and services. The Zachman framework is the oldest of these models and that was originally developed for the US department of defense. Zachman compared the framework with the one followed in the construction industry and developed the following empirical model: The following Figure 1 illustrates the Zachman model.

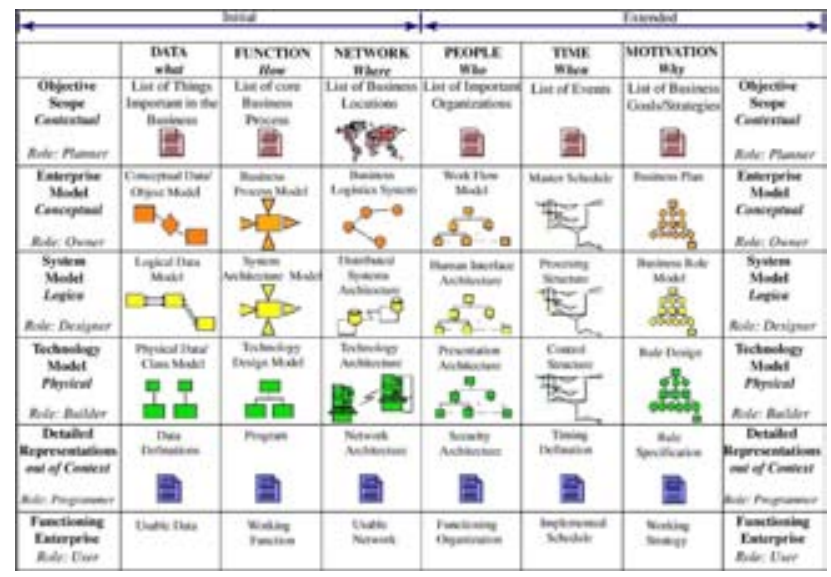

Fig. 1. The Zachman's Framework for Enterprise Architecture 
A close look of the model reveals that Zachman has tried to map the view of IT modelling from the perspective of people in various roles in the organisation. Noran (2003) mapped the Zachman's framework with the life cycle phases of GERA to evolve the following model illustrated in Figure 2:

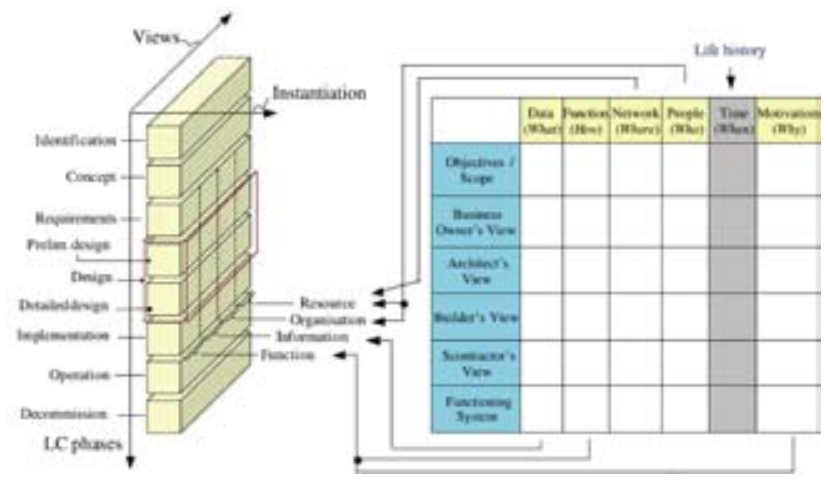

Fig. 2. Mapping of GERA with Zachman's model (Source : Noran 2003)

A closer analysis of the two models reveals that the real challenge for IT architects is to integrate all the viewpoints to develop the single large services framework that shall support the business framework of organisations. The cloud computing model is such a services framework that integrates all such viewpoints to develop a large, integrated infrastructure best suited for business needs. The people with various roles working on the platform will view cloud computing assisting their business needs from their own perspective. For example, the end users will view it from the perspective of their applications; the in-house IT management team will view it from the perspective of a set of virtual machine services purchased from the cloud hosting service provider. The hosting service provider will view it from the perspective of a number of server systems running hypervisors to support multiple virtual servers running over the top of the hypervisors. This mapping shall develop a model for cloud computing that may bring it closer to other computing models popular in the IT industry.

We present a mapping of cloud computing with respect to IT services and governance models focusing on the fact that from a business perspective the cloud computing is more of a services framework than an infrastructure. The most popular model for IT services in the industry is the ITIL framework which is a combination of two sets of best practices - service support and service delivery (www.itil.org). The Service Support framework comprises of a service desk controlling five critical service procedures - Incident Management, Problem Management, Change Management, Release Management and Configuration Management. The procedures help the IT services team to deliver business enabling tasks to ensure that the IT systems deliver optimum uptime and performance to the business. If we map the cloud computing framework to ITIL, a number of processes shall be established against the service procedures controlled by the service desk which in turn will have complete visibility into the entire computing environment by virtue of access to the component owners of the computing infrastructure - such as hardware, hypervisors, virtual machines, networks, applications, user profiles, authentication, authorisation and accounting for security. The procedures under service delivery comprises of service level, capacity, availability, financials and Business
Continuity Planning management which again will have specific sets under the cloud computing environment. As an example, the service level management shall comprise of a set of SLAs signed with the service provider that hosts the virtual machines for corporations [www.itil.org].

COBIT (Control Objectives for Information and related Technology) is a much larger framework compared to ITIL given that it is designed to implement the entire IT governance and not only the services. The COBIT framework provides the control objectives and controls in a logically organised structure comprising of best practices designed by a panel of experts. The primary objective of this framework is to link IT objectives with business requirements such that a process model for IT activities can be designed to ensure optimum utilisation of the major IT resources with respect to IT management control objectives. The model is shown in Figure 3:

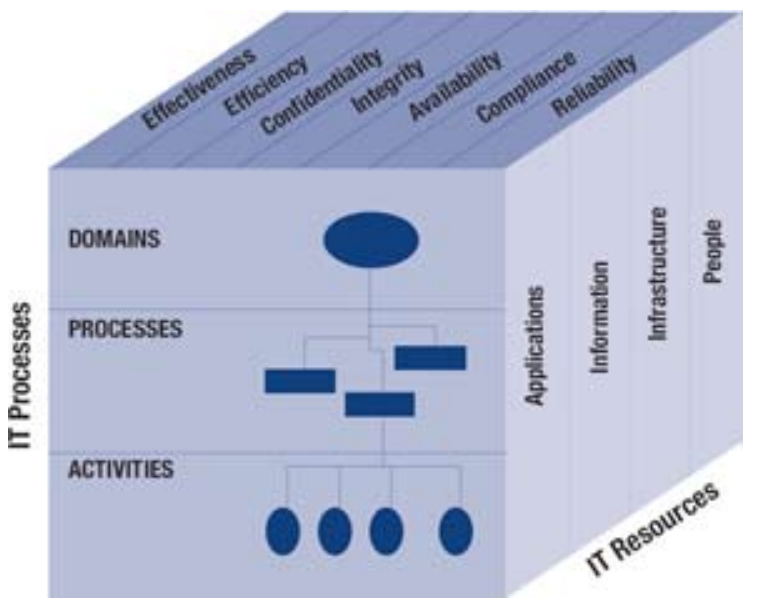

Fig. 3. The Cobit Framework (Source: COBIT 4.1 Executive Summary, ISACA.org, 2007)

The framework shows mapping of IT processes and IT resources with respect to the key objectives for the business effectiveness, efficiency, confidentiality, integrity, availability, compliance and reliability [ISACA.org 2007]. In the context of cloud computing, such a mapping shall be between the IT processes for the business (such as ERP support processes) with the cloud computing resources (such as hosting service providers, cloud computing infrastructure, distribution of applications in virtual machines etc.). The mapping of cloud computing with these models points towards the need for a dedicated enterprise architecture for cloud computing. Is there any enterprise architecture that is already modeled?

\subsection{Is there an Enterprise Architecture for Cloud Computing?}

In research literature there is little report on efforts to develop enterprise architecture for cloud computing and hence an objective of this paper is to discuss the characteristics of an enterprise architecture model for cloud computing. Buyya \& Yeo et al. (2008), developed a market oriented architecture for cloud computing that can be developed into an enterprise architecture model for cloud computing after combining with the relevant excerpts of other established enterprise architecture models.

The market oriented model of cloud computing is presented in Figure 4 below. 


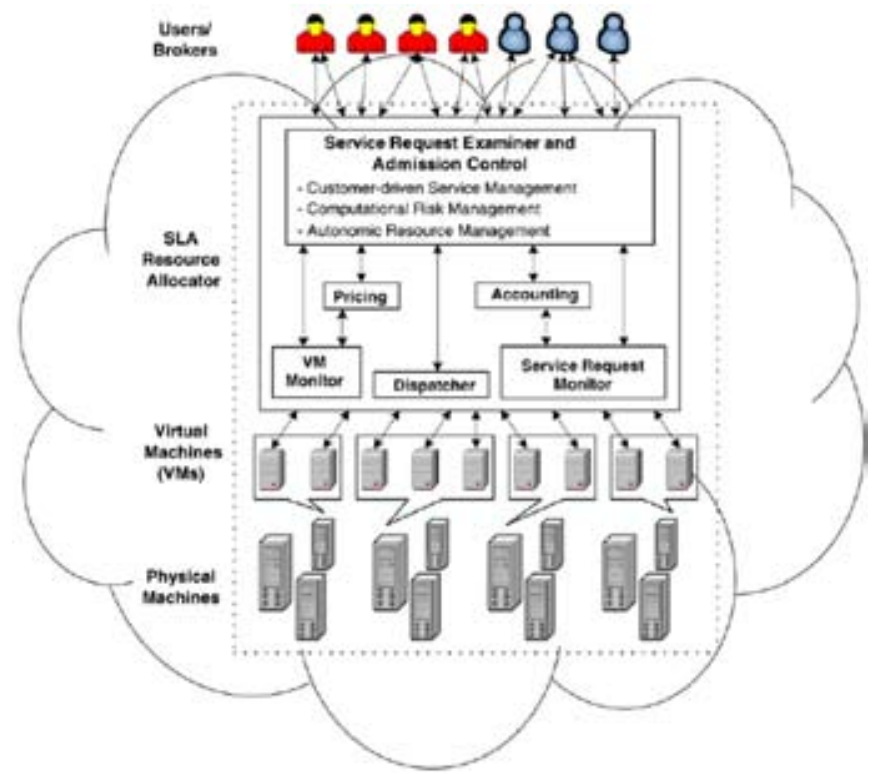

Fig. 4. An enterprise architecture for cloud computing (Source : Buyya and Yeo et al. 2009)

It is important to understand the thought process and philosophy of the model developed by Buyya \& Yeo et al. (2008 \&2009). They are the first few that have tried to develop a model for cloud computing. They have designed four layers in their model - the user layer, SLA resource allocation layer (includes accounting and billing as well), the virtualization layer and the physical machines layer. The service design is the key feature of this model whereby the authors have modeled a resource allocation system against service requests whereby the utilisation of resource can be tracked, accounted and billed as per a cost plan. As an example, we are using MS Word on a standard desktop consuming single dedicated Microsoft Office license. If we apply the same scenario in a virtualised environment, we may put a request for MS Word which is then procured from the cloud such that the usage is billed as per their billing plan for MS Word resource

\section{PROPOSED CLOUD COMPUTING TIPPING POINT MODEL}

The most popular model that defines a large effective framework for IT enablement of business is the COBIT that we discussed earlier. Most of the organizations are migrating to IT enabled businesses and hence the components of IT systems and applications have gradually achieved the position of being most valuable assets of the organisation. In this context the companies having high dependence on IT need to practice IT Risk Management to comply with regulatory requirements and manage the business dependence on IT effectively and lawfully. Hence, it is mandatory that IT Management and Governance becomes the direct responsibility of the board of directors and underlying top management layer of an organization. The advantages of having well managed IT Governance in organizations include the following: (ISACA. org 2007) (a) Effective management of business assets

(b) Sound fundamentals for the Enterprise Governance with effective Risk Management

(c) Optimized usage of the available IT resources

(d) Better threat management

(e) Capitalising of market opportunities

(f) Improved competitive advantages in the target markets

(g) Better compliance to laws and regulations

(h) Improved business performance management

(i) High customer satisfaction

(j) Faster growth

(k) Knowledge retention and management

(l) Objective management using KPI (key performance indicators) based approach]

Modern ICT organizations need to justify their cost by ensuring optimal return on investment given the high dependence of businesses on IT. They are very closely monitored by the top management and board members. Key IT issues now find enough priority in the board level discussions and hence modern IT organizations can no longer only be a group of technical experts. The objectives of modern ICT organization are defined by the COBIT objectives model presented in the below Figure 5 .

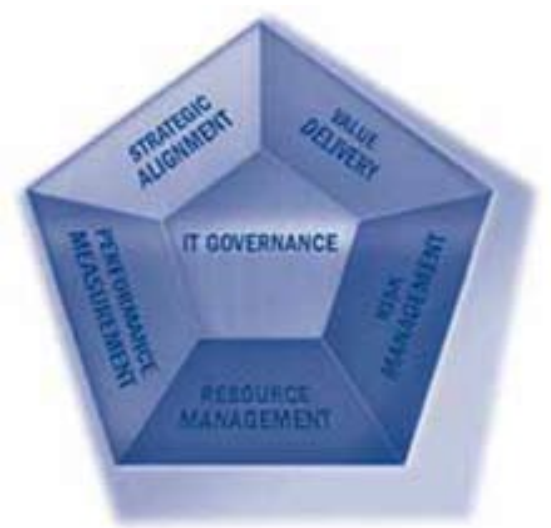

Fig. 5. CoBIT focus areas (Source: ISACA.org. 2007)

The modern IT management requires to focus on strategic alignment of IT objectives with business objectives, ensure high value delivery to the business, ensure that all IT resources (that is, people, processes and technologies) are managed effectively, and enable risk management and objective performance management. The most important process area is performance management of IT by setting measurable objectives pertaining to what the IT systems and processes are expected to deliver to the business. These processes need to be monitored closely using up to date continuous auditing tools. Thus transparency of internal processes and their deliverables is the key aspect of IT organizations in the modern business world.

We believe these attributes plays a major role in cloud computing enterprise architecture. Therefore, a model based on these attributes will be able to define characteristics of cloud computing artifact as opposed to in house hosted artifact. We believe by anylysing these attributes further, we can build a model that can advise organisations on their IT investment in 
relation to leveraging either cloud computing model or in house hosting model. Therefore, for an example, an organisation can be able to make a decision at a given point of time whether they will continue to procure new hardware and software from traditional vendors or do they subscribe to cloud computing hardware and software offerings. We define this equilibrium point as the "Cloud Computing Tipping Point". Let's discuss whether we can leverage this information to define attributes of cloud computing tipping point model.

\subsection{Cloud Computing Tipping Point Model}

The authors are exploring whether they could use COBIT as a model to define the attributes for the Cloud Computing Tipping Point model. The authors believe due to the high usage of COBIT in the industry and proven control structures will assist to build a solid foundation for their Tipping Point model. The outcome of this paper shall be a COBIT enabled cloud computing model clearly indicating the various roles contributing to the maturity of the model. The primary attributes planned for the model are:

(a) Shorter implementation life cycles of IT systems \& applications

(b) Enhanced service levels

(c) Higher uptime

(d) Better business continuity

(e) Excellent capacity \& availability management

(f) Low cost operations

(g) Better risk management \& information security

(h) Better software development life cycle management

We investigated the COBIT control objectives in detail to extract the most relevant control objectives that related to cloud computing. They have concluded the following control objectives stands out from the rest.
(a) Acquire and Maintain Application Software
(b) Procure IT Resources
(c) Acquire and Maintain Technology Infrastructure.
(d) Install and Accredit Solutions and Changes
(e) Manage Performance and Capacity
(f) Define and Manage Service Level
(g) Ensure Continuous Service

We also categorized COBIT control structures to quantitative financial attributes and qualitative attributes. The quantitative information could be gathered by investigating past and present financial information (i.e. Return on Investment, Total Cost of Ownership etc.). The qualitative information needs to be captured by a specialized tool created as a result of this exercise.

The authors also eventuated that some of the long term strategic objectives of a Cloud solution is not addressed by the COBIT control structures. Therefore, they have leveraged ValIT model to address the return on Investment, Internal Rate of Return and Total cost of ownership strategic insight. Val IT addresses assumptions, costs, risks and outcomes related to a balanced portfolio of IT-enabled business investments. It also provides benchmarking capability and allows enterprises to exchange experiences on best practices for value management. (ValIT 2010). Therefore, the authors have decided to leverage CoBIT and ValIT to provide the governance framework for Cloud Computing Tipping Point model.

After evaluating all these observations, the authors have created the following Cloud Computing Tipping Point model. The authors believe this model will assist IT organization to discover the "tipping point" of leveraging Cloud platforms from their existing in house hosting models. The model takes into account both quantitative and qualitative information to educate the users the suitability of investing the resources in relation to cloud computing as opposed to in-house hosting. The following conceptual model describes the Cloud Computing Tipping Point Model.

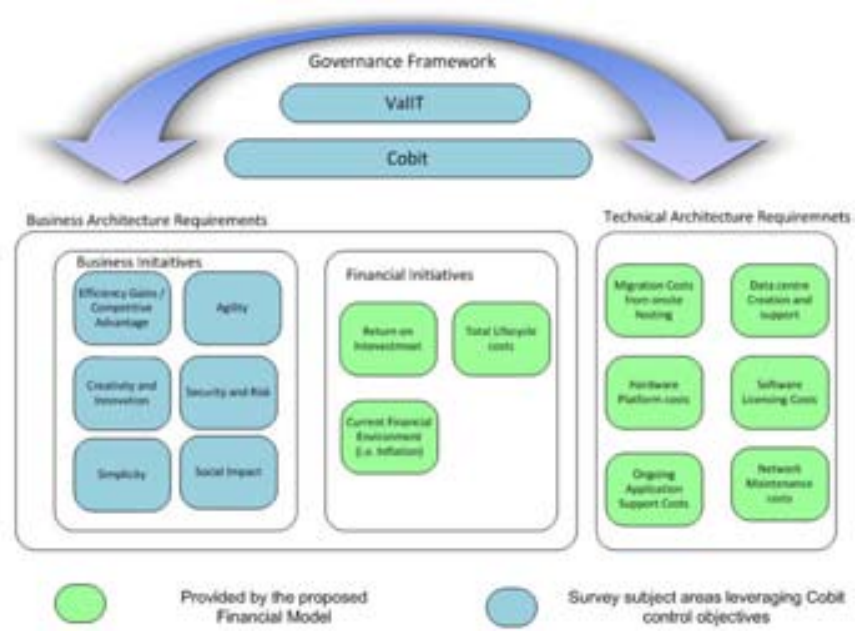

Fig. 6. Conceptual Cloud Computing Tipping Point Model

The authors believe the models comprises of two architecture streams. They are Business architecture and Technical architecture. (Peiris et al 2010)

\subsubsection{Business Architecture Attributes}

The model is based on two primaries objectives. They are business objectives and financial objectives. The business objectives comprises of:

(a) Efficiency Gains and resulting increase in Competitive Advantages

(b) Improved agility

(c) Better creativity and innovation in products \& customer services

(d) Better security and risk management 
(e) Simplicity of IT systems

(f) Better socialization among employees

The financial objectives comprises of:

(a) Better return on investment in IT

(b) Improvement in total lifecycle costs of IT deliverables

(c) Reduction in ongoing recurring costs - like support expenses

(d) Better response to financial distress or economic slowdown conditions.

\subsubsection{Technical Architecture Attributes}

The IT needs to respond to these business and financial objectives by ensuring that is a reduction in costs of:

(a) Data centres creation, running and maintenance

(b) Hardware platforms cost

(c) Software licensing cost

(d) Application support cost

(e) Network management cost

These cost reductions can be achieved by transition of IT infrastructure from on-site to virtualized hosting which will have its own costs. Hence, the ROI needs to be worked out very clearly that shall tradeoff the one time transition costs.

The authors have proceeded to validate the model by creating a logical model of the conceptual model. The authors believe the ValIT and high level COBIT control structures provide the governance framework for the model. The authors propose to implement a financial model for quantitative analysis. The quantitative analysis can be carried out on the following attributes.

(a) Better return on investment in IT

(b) Improvement in total lifecycle costs of IT deliverables

(c) Reduction in ongoing recurring costs - like support expenses

(d) Data centres creation, running and maintenance

(e) Hardware platform cost

(f) Software licensing cost

(g) Application support cost

(h) Network management cost

The authors also recognise that there are qualitative variables in their Tipping Point model. They propose to validate these qualitative variables by leveraging a series of survey questions. These qualitative variables are, (a) Efficiency Gains and resulting increase in Competitive Advantages

(b) Improved agility

(c) Better creativity and innovation in products \& customer services

(d) Better security and risk management

(e) Simplicity of IT systems

(f) Better socialization among employees

The following Figure 7 illustrates the Tipping Point logical architecture that differentiates the quantitative and qualitative attributes.

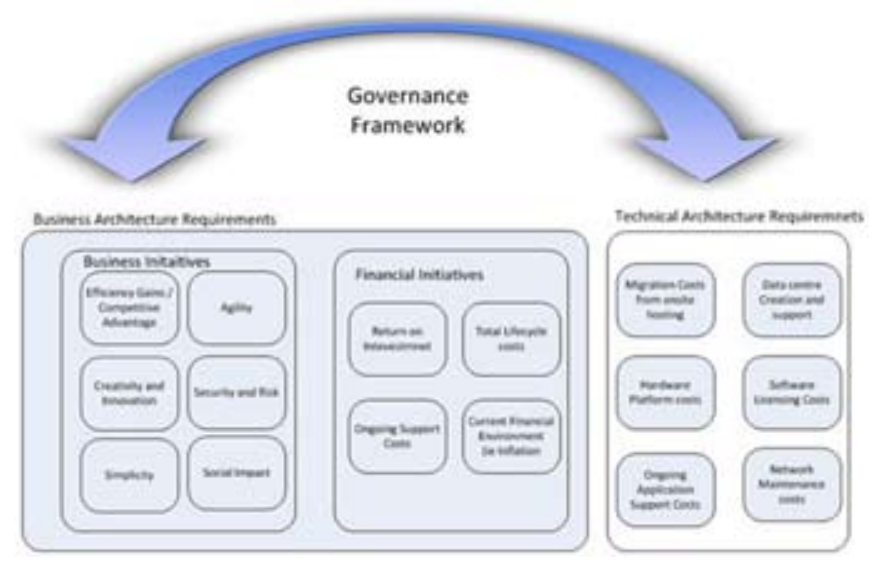

Fig. 7. Logical Cloud Computing Tipping Point

The authors are currently validating the above model by conducting a survey among users and non-user of Cloud Computing. The survey was conducted online my targeting technical and business users that represent both large enterprises and small SME organisations. The following Figure 8 illustrates the survey content.

The survey commences with a data input screen for prospective survey users. The objective of this screen is to set the valid context for the survey collect the information regarding

(a) What cloud computing domain does the user belong to? (i.e. email hosting, infrastructure hosting, application hosting etc.)

(b) Is the user leveraging cloud computing in his / her daily activities

(c) Is the user and business user or a technical user

(d) What organisation do the users represent (i.e. enterprise of SME organization.)

The user is presented with series of questions regarding his / her experience with Cloud Computing interactions. The samples survey questions are 
similar to Figure 8. The user will answer approximately 50 questions to provide data that is related to Cloud Computing Tipping model. The objective of the model is to calculate the suitability of leveraging the cloud platform (as opposed to in house hosting model) as a result of the answers provided by the user.

The authors are currently fine tuning and expanding both the qualitative and quantitative attributes of the model. They are hoping to expose this model as a set of services as the eventual artifact. Hence, the authors refer to this model as "Service Centric Cloud Computing Tipping Point Model".

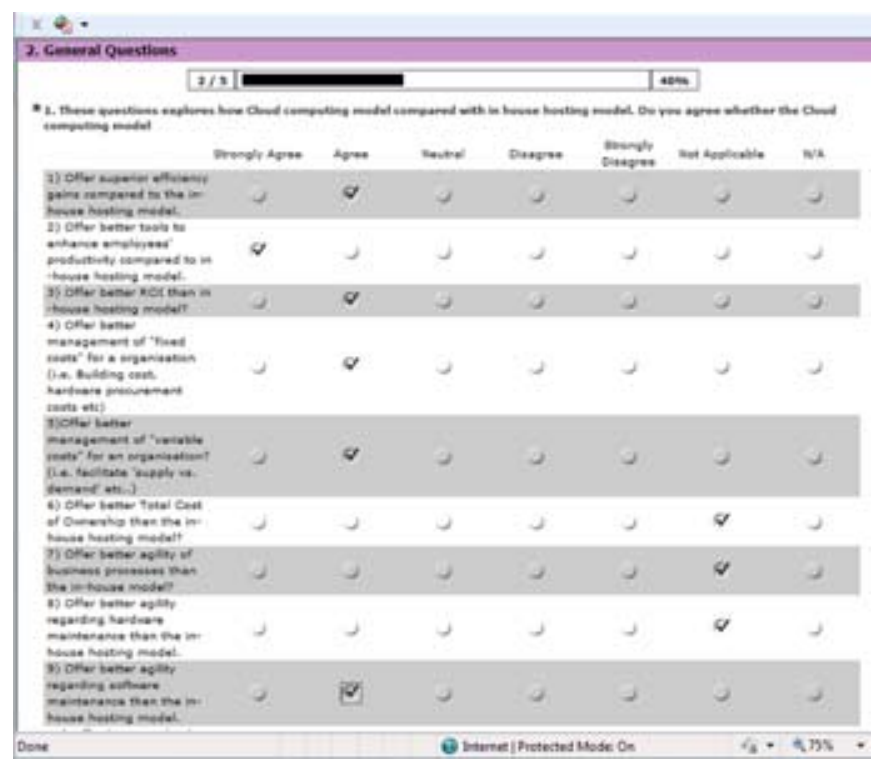

Fig. 8. Survey to validate findings - Survey pages

\section{CONCLUSIONS AND FUTURE WORK}

The major hosting providers have launched application programming interfaces for development of cloud friendly programs that can be run in Software as a Service mode. The current hosting providers have kept the backend architectures proprietary and hence there is a risk of the applications getting tied to specific cloud computing environments. The current researchers feel a need for standard cloud infrastructure and an enterprise model that all hosting providers should follow in order to ensure openness of the system from customer perspective. The author of this paper agrees with these arguments and shall contribute to such an open architecture model supporting the empirical generalisations being attempted by the current researchers.

We propose the Cloud Computing Tipping Point model that can assist an ICT organisation to understand their "readiness" or evaluate the investment they would like to make into the cloud offerings. The authors believe the Tipping Point model that is based on both quantitative and qualitative data will enable organisations to make rational and realistic decisions to invest either in cloud computing offerings or in house hosting options. We are also currently attempting to expose this capability as a service centric model for organization to evaluate their "readiness" to cloud computing offerings. We believe by facilitating this important toolset to the industry we are actively contributing to cloud computing enterprise architecture

Currently the authors are continuing with the surveys to evaluate this model and refine the model according to the feedback from industry and researchers. The authors are planning to create a service centric online model / artifact as the end deliverable as future work

\section{REFERENCES}

[1] Armbrust, Michael and Fox, Armando et al. (2009). Above the Clouds: A Berkeley View of Cloud Computing. Technical Report No. UCB/ EECS-2009-28. Electrical Engineering and Computer Sciences. University of California at Berkeley: 3-25.

[2] Buyya, Rajkumar and Ranjan, Rajiv et al. (2009). Modeling and Simulation of Scalable Cloud Computing Environments and the CloudSim Toolkit: Challenges and Opportunities. Grid Computing and Distributed Systems (GRIDS) Laboratory. Department of Computer Science and Software Engineering. The University of Melbourne, Australia: 1-11.

[3] Buyya, Rajkumar and Yeo, Chee Shin et al. (2009). Cloud computing and emerging IT platforms: Vision, hype, and reality for delivering computing as the 5th utility. Future Generation Computer Systems. Elsevier and Science Direct. Vol. 25: 599-616.Tavel, P. 2007 Modeling and Simulation Design. AK Peters Ltd.

[4] Buyya, Rajkumar and Yeo, Chee Shin et al. (2008). Market Oriented Cloud Computing: Vision, hype, and reality for delivering IT Services as Computing Utilities. Grid Computing and Distributed Systems (GRIDS) Laboratory.. The University of Melbourne, Australia, J. Mach. Learn. Res. 3 (Mar. 2003), 1289-1305.

[5] COBIT 4.1 - Executive Summary Framework. IT Governance Institute. ISACA.org. 2007: 4-16

[6] Noran, Ovidiu. (2003). An analysis of the Zachman framework for enterprise architecture from the GERAM perspective. Annual Reviews in Control. Pergamon, Elsevier. Science Direct. 27: 163-183.

[7] Peiris, Balachandran, Sharma (2010)), Cloud Computing Value Proposition: An Interrogation; Cloud Computing \& Virtualization, Singapore, 6-7

[8] Sarbanes Oxley Act of 2002. H.R.3763. Findlaw.com. 2002

[9] ValIT, ISACA (2010), http://www.isaca.org/Template. cfm? Section=Val_IT3\&Template=/TaggedPage/TaggedPageDisplay. cfm\&TPLID $=80 \&$ ContentID $=51867$ : page 1

[10] Virtualization Overview. VMWare and Global Knowledge Expert Reference Series of White Papers. VMWare Inc. 2008: 2-14

[11] Yang, C. \& Huang, J. (2000). A decision model for IS outsourcing. International Journal of Information Management, 20 (3): 225-239

[12] Zachman, J.A. (1987). A framework for information systems architecture. IBM Systems Journal. 26 (3): 1-17.

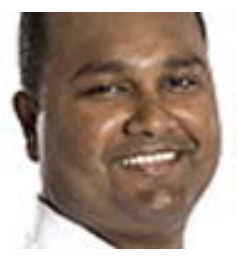

Chris Peiris is a frequent speaker at academic and industry conferences. Chris was a senior research fellow and a lecturer at Monash University, VIC, Australia. He currently guest lectures at University of Canberra, ACT, Australia. Chris has written many books, articles, reviews, and columns for various publications. His book titles include Security, Web services, UDDI, WCF, C\#, IIS, and Java subject areas, His complete list of publications and contact details are available at http://www.chrispeiris.com. 